\title{
Generalizing the Apple-Psych system
}

\author{
GIL OSGOOD \\ University of Oregon, Eugene, Oregon
}

\begin{abstract}
Each upgrade of computer hardware usually requires the development of new programming systems for psychological experimentation. Developing new software means learning a new system and unlearning an old one. To minimize this problem, a set of subroutine calls, derived from the Apple-Psych system, can be implemented with different dialects of Pascal on different microcomputers.
\end{abstract}

Psychologists first started using dedicated laboratory computers for experimental control in the early 1960s. Since then, dozens, if not hundreds, of systems have been developed in an attempt to simplify this task. The current issue of Behavior Research Methods, Instruments, \& Computers contains descriptions of several new systems (Costin, 1988; Poltrock \& Foltz, 1988; Schneider, 1988). During the last 20 years, I have written systems for the PDP-9, PDP-15, Prime 300, Apple II+, Apple Ile, and PDP-11-83,84 computers (Lewis, Boies, \& Osgood, 1971; Osgood, 1973, 1982).

The purpose of all this software has been to provide a powerful, yet easy to use, interface to the computer for the experimental psychologist. These systems have improved as better hardware and better programming tools have become available, but they continue to suffer from a critical flaw. Because of the real-time requirements of psychological experiments, these systems are tightly linked to specific types of computers that are equipped with specific peripherals. If there is a different kind of clock card or a new computer model, then the software no longer works. This is costly, both because of the continuing investment in program development and because of the need for the users of the software to keep unlearning old systems and learning new ones. The problem has become more acute as microcomputers have made computing available to smaller psychology departments that cannot afford professional programmers and hardware technicians.

The ideal system would be easily transported to new hardware while maintaining a stable interface to the user. The fundamental problem, of course, is that computer hardware is continuing to undergo rapid change with little standardization. The situation is analogous to the early days of automobiles, when the driver steered the steam, electric, or gas-powered vehicle with a rudder or steering wheel or foot pedals from the left, right, or center of the front seat. The central thesis of this paper is that a partial solution to the goal of a transportable system for experiment control does not have to await the standardization of hardware designs.

Address all correspondence to Gil Osgood, Department of Psychology, Straub Hall, University of Oregon, Eugene, OR 97405.

\section{THREE KINDS OF INTERFACES}

Every piece of software has two interfaces, one to the human being who uses it and one to the hardware on which it runs. The problem of transporting a piece of software to a new computer is one of adapting the hardware interface while preserving the user interface. A partial solution to the problem is to break the software into two pieces, a hardware-dependent part and a hardwareindependent part. Some Pascal compilers are implemented in this way. Portable code is written that translates Pascal programs into a kind of generalized machine language instead of the machine code of a particular computer. This abstract machine language is then executed by an interpreter written for the particular brand of computer. To transport the software, only the interpreter must be rewritten. This is much easier than rewriting an entire compiler. This approach does introduce a third interface, the one between the two parts of the software package, which must be carefully defined.

Software for the control of psychological experiments can likewise be broken down into two parts. The bulk of the code can be written in Pascal, a computer language that is available for most microcomputers. The code that is unique to a particular kind of computer with a particular set of peripherals can be written in Pascal or assembly language. To ensure portability, this code must provide a standard set of subroutine entries that can be accessed by the portable part of the system. This kind of system is like a two-layer cake. The bottom layer (the unique code) rests on a plate (the hardware). The top layer (the portable code) is the part that the consumer (the user) sees. This division between idiosyncratic and common code is partially implemented in the Apple-Psych

Table 1

The Six Basic Tasks in Psychological Experimentation

\begin{tabular}{ll}
\multicolumn{1}{c}{ Task } & Apple-Psych Library Unit \\
\hline Timing & OUTERWORLD \\
Stimulus Presentation & OUTERWORLD \\
Response Recording & OUTERWORLD \\
Stimulus Generation & TVSCREEN \\
Randomization & IORAND \\
Parameter Input & IORAND \\
\hline
\end{tabular}


system. The rest of this paper is devoted to describing a system, generalized from the Apple-Psych software, that embodies these ideas. The emphasis is on how to make the bottom layer (the unique code) as small as possible and on what the thin strip of icing that separates the two layers (the subroutine entries to the unique code) must look like.

\section{ELEMENTS OF EXPERIMENTAL CONTROL SYSTEMS}

Software for the computerized control of real-time psychological experiments, although often quite complex, can be described in terms of the six basic tasks shown in Table 1. The timing, stimulus presentation, and response recording tasks are the most critical from the perspective of the psychology of the experiment. They are also quite hardware dependent. Stimulus generation has less stringent timing requirements than do the first three tasks, but it is also very hardware dependent. The randomization and parameter input tasks are largely independent of the hardware. These six tasks are distributed among the library units that constitute the Apple-Psych system, as shown in Table 1. The unique code and the portable code for each of these tasks are described below. The key to this approach is to always make the unique code look the same to the portable code, regardless of the particular machine or peripherals involved. Consequently, in the discussion that follows, special emphasis is placed on describing the subroutine entries into the unique code.

\section{Timing}

Timing is the most idiosyncratic system component, due to the wide variety of hardware clocks. The clock is used for timing stimulus presentations and response latencies with millisecond accuracy. Some provision must also be made to compensate for the $50-$ or $60-\mathrm{Hz}$ refresh cycles of the TVs and monitors that are the most common stimulus presentation devices. The following subroutine entries into the unique code satisfy these requirements:

1. INITCLOCK-performs initialization of the clock, such as setting up interrupt vectors if needed.

2. WAITSYNCH (position)-waits until the raster scan refresh cycle has reached a specified position.

3. STARTCLOCK - starts the clock either by issuing the hardware start command or by reading the current time.

4. READCLOCK (time)-reports the time elapsed since the STARTCLOCK to the calling program.

5. STOPCLOCK (time)-stops the clock and reports the elapsed time.

The portable code can lock the timing of an experiment trial to the monitor refresh cycle by doing a WAITSYNCH before calling STARTCLOCK. The READ-
CLOCK is used to monitor the time while preparing for stimulus presentation or response collection.

\section{Stimulus Presentation}

A variety of devices can be used to present stimuli: the computer monitor, arrays of lights, inline displays, slide projectors, white noise generators, tactile vibrators, and so forth. The vast majority of this confusing collection of equipment is activated simply by throwing switches (i.e., digital devices). A few devices require A/D conversion, but they will be ignored for the purposes of this discussion. In particular, computer screens can be treated as digital devices. This is because the analog raster scan, which refreshes the screen image, proceeds automatically, getting its information from a particular area of the computer's memory. Generally, there are several of these screen memories. Stimuli can be turned on and off simply by switching from one screen memory to another. The portable code operates by placing switching requests for any device into a queue. Information in this list includes the device identification, the switch or switches to be thrown, and the time at which the switching should take effect. Only two unique code subroutines are needed:

1. INITSTIM-initializes stimulus devices.

2. GETSTIM-retrieves a switch request from the head of the queue and executes it.

During the time-critical portion of the experiment trial, the portable code calls READCLOCK to check the time and compares it with the time of the first switching event in the queue. When switching becomes due, a call is made to the unique code procedure GETSTIM to do the actual switching.

\section{Response Recording}

Most response recording is a matter of detecting switch closures (the special case of acquiring analog data is not considered here). As with stimulus presentation, a queue is used. The unique code must contain a subroutine (PUTRESP) that scans response devices and places device identification, key codes, and response times into the queue when a response is made. The portable code includes a subroutine that retrieves responses from this queue and checks them to see if they came from the appropriate device during the appropriate response period. The following unique code subroutines are needed:

1. INITRESP-initializes response devices.

2. PUTRESP - saves response information in the response queue.

\section{Stimulus Generation}

The creation of stimuli prior to presentation is the most hardware-dependent part of the system because stimulus devices vary so much. We consider here only the problem of setting up the stimuli in the screen memories, since the computer screen is the most common stimulus device. 
The basic unique code functions required are clearing the screen memory, writing text strings into it, and drawing figures in it:

1. CLEARSCREEN (screen memory)-erases the selected screen memory.

2. DRAWTEXT (screen memory, text string, space, line)-writes a text string at the specified location in the selected screen memory.

3. DRAWFIG (screen memory, figure string, $x$ coordinate, $y$ coordinate)-draws a simple figure at the specified location in the selected screen memory. The string variable contains instructions for drawing the figure.

Global variables can be used to assign other display attributes, such as character size and orientation, spacing, and background and foreground color. The portable code can combine these subroutine calls with the stimulus presentation and timing calls to set up stimuli and schedule their display and deletion.

\section{Randomization}

In general, the portable code can contain all the randomization routines needed for running experiments. These include the basic random number generator and routines that use it to randomly select experimental conditions and to shuffle stimulus arrays. The crucial feature to check when transporting code is the algorithm for generating pseudorandom numbers. If the size of an integer variable changes (e.g., from 16 to 32 bits), the cycle of the random number generator may change or the algorithm may be affected in more subtle ways.

\section{Parameter Input}

To ensure system integrity, all parameter input should be done through a standard set of routines. These procedures must ask a question, provide some clues as to what constitutes a legal answer, check the answer, and, if it is not suitable, reask the question. For example, timing parameters relating to stimulus presentations on the monitor screen must be in multiples of the refresh cycle (i.e., in multiples of $16.67 \mathrm{msec}$ ). This can all be done in the portable code. The only unique code needed is a procedure to position the cursor on the text screen. This is usually built into the operating system, but the name may change from system to system. Thus, minor changes to the portable code may be necessary.

\section{CONCLUDING REMARKS}

Some additional reduction of the unique code may be possible by using a configuration file. For example, the problem of detecting the vertical position of the raster scan is usually a matter of testing a particular address in the computer. Although the address differs with different brands of computers, the test is the same. A configuration file containing the unique address could be read by the portable code.

Differences in implementations of Pascal may cause some other problems when transporting the system. For example, with UCSD Pascal, library files can be used to invoke the experiment control software, whereas Turbo Pascal requires the use of inline commands. Unlike UCSD Pascal, Turbo Pascal does not have a long integer type. This means that on 16-bit machines, integers can only be 16 bits wide, which means that times can accumulate to just over $32 \mathrm{sec}$. This type of problem is annoying but usually quite easy to overcome.

This paper is intended only as an introduction to the topic of stabilizing experiment software. A more rigorous and comprehensive definition of the interface between the unique code and the portable code is needed. Regardless of any limitations this general outline may have, the fact remains that we need to pay attention to the foundations upon which our psychological software is built and not simply to the imposing edifices that arise from them.

\section{REFERENCES}

Costin, D. (1988). MacLab: A Macintosh system for psychology labs. Behavior Research Methods, Instruments, \& Computers, 20 , 197-200.

LEWIS, J. L., BoIes, S. J., \& OsGood, G. W. (1971). Zoraster: A multiprogramming system for psychological research. Behavior Research Methods \& Instrumentation, 3, 106-107.

OsGoOD, G. W. (1973, August). The Pleiades time-sharing system. Paper presented at the Advanced Research Projects Agency Conference on Computers in Psychology, Montreal.

OsGooD, G. W. (1982). Apple-Psych programming guide (Coglab Rep. No. 6). Eugene, OR: University of Oregon, Center for Cognitive and Perceptual Research, Department of Psychology.

Poltrock, S. E., \& Foltz, G. S. (1988). APT PC and APT II: Experimental development systems for the IBM PC and Apple II. Behavior Research Methods, Instruments, \& Computers, 20, 201205.

SCHNEIDER, W. (1988). Micro Experimental Laboratory: An integrated system for IBM PC compatibles. Behavior Research Methods, Instruments, \& Computers, 20, 206-217. 